\title{
A Fuzzy Optimization Method for Multi-criteria Decision- making Problem Based on the Inclusion Degrees of Intuitionistic Fuzzy Sets
}

\author{
Yan Luo ${ }^{1}$ and Changrui $\mathrm{Yu}^{1,2+}$ \\ ${ }^{1}$ Institute of System Engineering, Shanghai Jiao Tong University, Shanghai 200052, China \\ ${ }^{2}$ School of Information Management and Engineering, Shanghai University of Finance and Economics, \\ Shanghai 200433, China
}

(Received January 18, 2007, accepted April 22, 2007)

\begin{abstract}
This paper presents a fuzzy optimization method based on the inclusion degrees of intuitionistic fuzzy sets to solve multi-criteria decision making problems under fuzzy environments. First, the inclusion degree of intuitionistic fuzzy sets is defined and a series of specific formulas of inclusion degree are presented by means of the normal implication operators. Some formulas of inclusion degree of intuitionistic fuzzy sets are generalized by defining the cardinal number of intuitionistic fuzzy sets. Then, we give multicriteria fuzzy decision-making method based on inclusion degree of intuitionistic fuzzy sets. Finally, we illustrate the effectiveness of the method proposed in this paper by an example.
\end{abstract}

Keywords: Multi-criteria Decision-making, Fuzzy Optimization, Inclusion Degrees, IFS.

\section{Introduction}

Fuzzy sets were introduced by Zadeh in 1965 [1]. In the following several decades, fuzzy set theory has been used for handling fuzzy multi-criteria decision-making problems [2-5]. The main characteristic of fuzzy sets is that: the membership function assigns to each element $u$ in a universe of discourse a membership degree ranging between 0 and 1 and the non-membership degree equals one minus the membership degree, i.e., this membership degree combines the evidence for $u$ and the evidence against $u$. The single number tells us nothing about the lack of knowledge. In real applications, however, the information of an object corresponding to a fuzzy concept may be incomplete, i.e., the sum of the membership degree and the nonmembership degree of an element in a universe corresponding to a fuzzy concept may be less than one. In fuzzy set theory, there is no means to incorporate the lack of knowledge with the membership degrees. A possible solution is to use intuitionistic fuzzy sets (IFSs for short), introduced by Atanassov in [6]. IFS as an extension of Zadeh fuzzy set was also applied to the decision-making problems [7-12].

In the present paper, the inclusion degree of intuitionistic fuzzy sets is applied to multi-criteria decision making in fuzzy environment. In Section 2 we define the inclusion degree of intuitionistic fuzzy sets and present a series of specific formulas of inclusion degree by means of the normal implication operators. Then some formulas of inclusion degree of fuzzy sets are generalized to intuitionistic fuzzy sets by defining the cardinal number of intuitionistic fuzzy sets. In Section 3 we give a fuzzy method for multi-criteria decisionmaking method based on inclusion degree of intuitionistic fuzzy sets. Finally, we illustrate the effectiveness of the method proposed in this paper by an example.

\section{Inclusion degrees of IFSs}

The concept of IFSs is an extension of Zadeh's fuzzy sets. It give us the possibility to model unknown information by using an additional degree. In [6] [13,14], IFSs are defined as follows:

Definition 1. An intuitionistic fuzzy set $A$ on a universe $U$ is defined as an object of the following form:

\footnotetext{
${ }^{+}$Corresponding author. Tel.: +86-21-62811769; fax: +86-21-52301193.

E-mail address: yucr@sjtu.edu.cn.
} 


$$
A=\left\{\left(u, \mu_{A}(u), v_{A}(u)\right) \mid u \in U\right\},
$$

where the functions $u_{A}: U \rightarrow[0,1]$ and $v_{A}: U \rightarrow[0,1]$ define the degree of membership and the degree of non-membership of the element $u \in U$ in A, respectively, and for every $u \in U$ :

$$
0 \leq \mu_{A}(u)+v_{A}(u) \leq 1,
$$

Obviously, each ordinary fuzzy set may be written as $\left\{\left(u, \mu_{A}(u), 1-\mu_{A}(u)\right) \mid u \in U\right\}$. For simplicity, the class of intuitionistic fuzzy sets on a universe $U$ will be denoted by $\operatorname{IFS}(U)$.

\subsection{Inclusion degrees based on implicational operators}

Definition 2 If the mapping $I: \operatorname{IFS}(U) \times \operatorname{IFS}(U) \rightarrow[0,1]$ satisfies

(i) $A \subseteq B \Rightarrow I(A, B)=1$;

(ii) $I(U, \phi)=0$;

(iii) $A \subseteq B \subseteq C \Rightarrow I(C, A) \leq \min (I(B, A), I(C, B))$

then we call $I(A, B)$ the inclusion degree of $A$ in $B$, and call $I$ a inclusion degree function on $\operatorname{IFS}(U)$.

Definition 3 If mapping $R:[0,1]^{2} \rightarrow[0,1]$ satisfies

(i) $R(1,0)=0$

(ii) $R(0,0)=R(0,1)=R(1,1)=1$

then $R$ is called fuzzy implicational operator (briefly, implication).

Definition 4 If mapping $T:[0,1]^{2} \rightarrow[0,1]$ satisfies

(i) $T(a, b)=T(b, a)$

(ii) $a \leq c, b \leq d \Rightarrow T(a, b)=T(c, d)$

(iii) $T(T(a, b), c)=T(a, T(b, c))$

(iv) $T(a, 1)=a(a \in[0,1])$

then it is called triangular norm (briefly, $t$-norm). If $\mathrm{T}$ satisfies (i)-(iii) and (iv)' $T(0, a)=a(a \in[0,1])$, then we call it triangular conorm (briefly, $t$-conorm).

Theorem 1 Let $A, B \in \operatorname{IFS}(U)$ and $R$ be an implication. If $R$ satisfies

(i) $\forall a, b \in[0,1]$, and $a \leq b \Rightarrow R(a, b)=1$

(ii) $R(a, b)$ is non-decreasing with respect to $b$ non-increasing with respect to $a$, then the following $I_{1}, I_{2}$ are the inclusion degree functions of IFSs:

$$
\begin{gathered}
I_{1}(A, B)=\inf _{u \in U}\left[\lambda R\left(\mu_{A}(u), \mu_{B}(u)\right)+(1-\lambda) R\left(v_{B}(u), v_{A}(u)\right)\right], \lambda \in[0,1], \\
I_{2}(A, B)=\inf _{u \in U} T\left(R\left(\mu_{A}(u), \mu_{B}(u)\right), R\left(v_{B}(u), v_{A}(u)\right)\right)
\end{gathered}
$$

where $T:[0,1]^{2} \rightarrow[0,1]$ is a $t$-norm

Proof. We only prove $I_{2}$. The proof of $I_{1}$ is similar.

(i) $A \subseteq B \Rightarrow \mu_{A}(u) \leq \mu_{B}(u), v_{A}(u) \geq v_{B}(u), \forall u \in U$

$$
\begin{aligned}
& \Rightarrow R\left(\mu_{A}(u), \mu_{B}(u)\right)=1, R\left(v_{B}(u), v_{A}(u)\right)=1, \forall u \in U \\
& \Rightarrow I_{2}(A, B)=\inf _{u \in U} T(1,1)=1
\end{aligned}
$$

(ii) $I_{2}(U, \phi)=\inf _{u \in U} T(R(1,0), R(1,0))=\inf _{u \in U} T(0,0)=0$

(iii) $A \subseteq B \subseteq C \Rightarrow \mu_{A}(u) \leq \mu_{B}(u) \leq \mu_{C}(u), v_{A}(u) \geq v_{B}(u) \geq v_{C}(u)$

Since $R(a, \bullet)$ is non-decreasing and $R(\bullet, b)$ is non-increasing, we have 


$$
\begin{aligned}
& R\left(\mu_{C}(u), \mu_{A}(u)\right) \leq R\left(\mu_{C}(u), \mu_{B}(u)\right), R\left(v_{A}(u), v_{C}(u)\right) \leq R\left(v_{B}(u), v_{C}(u)\right) \\
\Rightarrow & T\left(R\left(\mu_{C}(u), \mu_{A}(u)\right), v_{A}(u), v_{C}(u)\right) \leq T\left(R\left(\mu_{C}(u), \mu_{B}(u)\right), R\left(v_{B}(u), v_{C}(u)\right)\right) \\
\Rightarrow & \operatorname{int}_{u \in U} T\left(R\left(\mu_{C}(u), \mu_{A}(u)\right), v_{A}(u), v_{C}(u)\right) \leq \operatorname{int}_{u \in U} T\left(R\left(\mu_{C}(u), \mu_{B}(u)\right), R\left(v_{B}(u), v_{C}(u)\right)\right) \\
\Rightarrow & I_{2}(C, A) \leq I_{2}(C, B)
\end{aligned}
$$

In a similar, we can get $I_{2}(C, A) \leq I_{2}(B, A)$.

Theorem 2 Assume that $U$ is a finite universe and $R$ is an implication. If $R$ satisfies

(i) $\forall a, b \in[0,1]$, and $a \leq b \Rightarrow R(a, b)=1$

(ii) $R(a, b)$ is non-decreasing with respect to $b$ non-increasing with respect to $a$, then the following $I_{3}, I_{4}$ are the inclusion degree functions of IFSs:

$$
\begin{gathered}
I_{3}(A, B)=\frac{1}{|U|} \sum_{u \in U}\left[\lambda R\left(\mu_{A}(u), \mu_{B}(u)\right)+(1-\lambda) R\left(v_{B}(u), v_{A}(u)\right)\right], \lambda \in[0,1], \\
I_{4}(A, B)=\frac{1}{|U|} \sum_{u \in U} T\left(R\left(\mu_{A}(u), \mu_{B}(u)\right), R\left(v_{B}(u), v_{A}(u)\right)\right)
\end{gathered}
$$

where $|U|$ denotes the cardinality of $U$ and $T:[0,1]^{2} \rightarrow[0,1]$ is a $t$-norm.

The proof of theorem 2 is similar to the proof of theorem 1.

The implications $R$ satisfy the conditions of above theorems, $\forall a, b \in[0,1]$ :

(i) Lukasiewicz implication: $R_{L}(a, b)=\min (1-a+b, 1)$

(ii) Goguen implication: $R_{\pi}(a, b)=\left\{\begin{array}{cll}1, & \text { if } & a=0, \\ \min \left(\frac{b}{a}, 1\right), & \text { if } & a>0 .\end{array}\right.$

(iii) Gödel implication: $R_{G}(a, b)=\left\{\begin{array}{lll}1, & \text { if } & a \leq b \\ b, & \text { if } & a>b\end{array}\right.$.

(iv) Gaines-Recher implication: $R_{G R}(a, b)=\left\{\begin{array}{lll}1, & \text { if } & a \leq b \\ 0, & \text { if } & a>b\end{array}\right.$

(v) $\mathrm{R}_{0}$-implication: $R_{\pi}(a, b)=\left\{\begin{array}{cll}1, & \text { if } & a \leq b \\ \max (1-a, b), & \text { if } & a>b\end{array}\right.$

\subsection{Inclusion degrees based on the cardinalities of sets}

\begin{tabular}{|c|c|c|c|c|c|}
\hline$I_{5}(A, B)=$ & $\left\{\begin{array}{c}1, \\
|A \cap B|\end{array}\right.$ & $\begin{array}{l}A=\phi \\
A \neq \phi\end{array} ;$ & $I_{6}(A, B)=$ & $\left\{\begin{array}{c}1, \\
\frac{|B|}{|A \cup B|},\end{array}\right.$ & $\begin{array}{l}A=B=\phi \\
\text { otherwise }\end{array}$ \\
\hline$I_{7}(A, B)=$ & $\begin{array}{c}1, \\
\left|A^{C} \cap B^{C}\right| \\
\left|B^{C}\right|\end{array}$ & $\begin{array}{l}B=U \\
B \neq U\end{array} ;$ & $I_{8}(A, B)=$ & $\frac{\left|A^{C}\right|}{\left|A^{C} \cup B^{C}\right|}$ & $\begin{array}{l}A=B=U \\
\text { otherwise }\end{array}$ \\
\hline
\end{tabular}

Definition 5 Let $U$ be a finite set, $A \in \operatorname{IFS}(U)$. The cardinality of $A$ is defined as

$$
|A|=\sum_{u \in U} \frac{1+\mu_{A}(u)-v_{A}(u)}{2},
$$

It is easy to prove that the following inclusion functions of fuzzy sets $I_{5}-I_{10}$ [15] still hold to IFSs.

Theorem 3 Let $U$ be a finite set, $A \in I F S(U)$. Then the following $I_{5}-I_{10}$ are inclusion degree functions of IFSs: 


$$
I_{9}(A, B)=\frac{\left|A^{C} \cup B\right|}{\left|A^{C} \cup A \cup B \cup B^{C}\right|} ; \quad I_{10}(A, B)=\left\{\begin{array}{cc}
1, & A=\phi \text { or } B=U \\
\frac{\left|A^{C} \cap A \cap B \cap B^{C}\right|}{\left|A \cup B^{C}\right|}, & \text { otherwise }
\end{array} .\right.
$$

\subsection{Generation of inclusion degrees}

Theorem 4 Let $I$ be an inclusion degree function on $\operatorname{IFS}(U)$ and mapping $h:[0,1]^{2} \rightarrow[0,1]$ satisfies

(i) $h(0,0)=0, h(1,1)=1$;

(ii) $h(a, b)$ is non-decreasing with respect to a and b

then $G I(A, B)=h\left(I(A, B), I\left(B^{C}, A^{C}\right)\right)(\forall A, B \in I F S(U))$ is inclusion degree of $A$ in $B$, and $G I$ is an inclusion degree on $I F S(U)$.

proof. (i) $A \subseteq B \Rightarrow I(A, B)=1, I\left(B^{C}, A^{C}\right)=1 \Rightarrow G I(A, B)=h(1,1)=1$

(ii) $G I(U, \phi)=h\left(I(U, \phi), I\left(\phi^{C}, U^{C}\right)\right)=h(0,0)=0$

(iii) $A \subseteq B \subseteq C \Rightarrow C^{C} \subseteq B^{C} \subseteq A^{C}$

$$
\begin{aligned}
& \Rightarrow I(C, A) \leq I(C, B), I\left(A^{C}, C^{C}\right) \leq I\left(B^{C}, C^{C}\right) \\
& \Rightarrow h\left(I(C, A), I\left(A^{C}, C^{C}\right)\right) \leq h\left(I(C, B), I\left(B^{C}, C^{C}\right)\right) \\
& \Rightarrow G I(C, A) \leq G I(C, B)
\end{aligned}
$$

In the similar way, we can get $G I(C, A) \leq G I(B, A)$

Theorem 5 Let $I_{1}, I_{2}$ be inclusion degree functions on IFS(U), and $h:[0,1]^{2} \rightarrow[0,1]$ satisfies

(i) $h(0,0)=0, h(1,1)=1$;

(ii) $h(a, b)$ is non-decreasing with respect to $a$ and $b$,

then $G I(A, B)=h\left(I_{1}(A, B), I_{2}(A, B)\right)(\forall A, B \in I F S(U))$ is inclusion degree of $A$ in $B$, and $G I$ is an inclusion degree on $\operatorname{IFS}(U)$.

The proof of theorem 5 is similar to the proof of theorem 4 .

\section{Multi-criteria fuzzy decision-making based on inclusion degrees}

Definition 6 (Multi-criteria fuzzy decision-making problem [7]). Let $M$ be a set of alternatives and let $C$ be a set of criteria, where

$$
M=\left\{M_{1}, M_{2}, \cdots, M_{m}\right\}, \quad C=\left\{C_{1}, C_{2}, \cdots, C_{n}\right\},
$$

Assume that the characteristics of the alternative $M_{i}$ are presented by the IFS shown as follows:

$$
M_{i}=\left\{\left(C_{1}, \mu_{i 1}, v_{i 1}\right),\left(C_{2}, \mu_{i 2}, v_{i 2}\right), \cdots,\left(C_{n}, \mu_{i n}, v_{i n}\right)\right\},
$$

where $\mu_{i j}$ indicates the degree to which the alternative $M_{i}$ satisfies criterion $C_{j}, v_{i j}$ indicates the degree to which the alternative $M_{i}$ does not satisfy criterion $C_{j}\left(\mu_{i j}, v_{i j}\right) \in L^{*}(j=1,2, \cdots, n ; i=1,2, \cdots, m)$.

Assume that there is a decision-maker who wants to choose an alternative which satisfies the criteria $C_{j}, C_{k}, \cdots$, and $C_{p}$ or which satisfies the criterion $C_{s}$. This decision-maker's requirement is represented by the following expression:

$$
C_{j} \text { and } C_{k} \text { and } \cdots \text { and } C_{p} \text { or } C_{s} \text {, }
$$

It is noted that we say an alternative satisfies a criterion if it meets some desirable level of an evaluation criterion. The satisfaction is gradual and is characterized by a dual information: a degree of satisfaction and a degree of non-satisfaction.

We now use the inclusion degrees of IFSs to solve the mufti-criteria fuzzy decision-making problem (definition in [7]).The basic idea is similar to the TOPSIS (Technique for Order Preference by Similarity to 
Ideal Solution) [16-20]. Firstly, the ideal solution and the anti-ideal solution are constructed, where the ideal solution and the anti-ideal solution are respectively the best and the worst solution supposed but not existing in the set of alternatives. Then we compare the inclusion degrees of the ideal solution in alternative $M_{i}$ and the inclusion degrees of alternative $M_{i}$ in the anti-ideal solution. The alternative containing the ideal solution maximally as well as being contained by the negatively ideal solution minimally is the best choice. For this purpose, we introduce the following definition.

Definition 7 Let $M_{i}=(i=1, \cdots, m)$ be the set of alternatives and $C_{j}=(j=1, \cdots, n)$ be the set of criteria,

(i) The ideal solution and the anti-ideal solution satisfying the criteria $C_{j}, C_{k}, \cdots C_{p}$ are defined as follows:

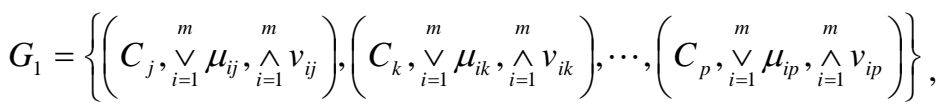

$$
\begin{aligned}
& =\left\{\left(C_{j}, \mu_{g j}, v_{g j}\right),\left(C_{k}, \mu_{g k}, v_{g k}\right), \cdots,\left(C_{p}, \mu_{g p}, v_{g p}\right)\right\}
\end{aligned}
$$

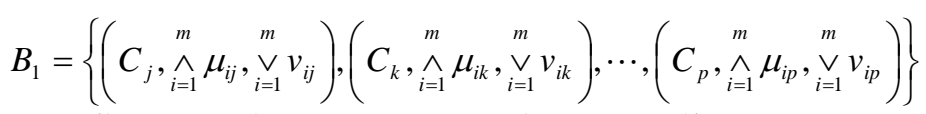

$$
\begin{aligned}
& =\left\{\left(C_{j}, \mu_{b j}, v_{b j}\right),\left(C_{k}, \mu_{b k}, v_{b k}\right), \cdots,\left(C_{p}, \mu_{b p}, v_{b p}\right)\right\}
\end{aligned}
$$

Definition 8 The inclusion degree $D\left(M_{i}\right)$ of the ideal solution in alternative $M_{i}$ and the inclusion degree $d\left(M_{i}\right)$ of alternative $M_{i}$ in the anti-ideal solution are respectively defined as follows:

$$
\begin{gathered}
D\left(M_{i}\right)=\max \left(I\left(G_{1}, M_{i 1}\right), I\left(G_{2}, M_{i 2}\right)\right), \\
d\left(M_{i}\right)=\min \left(I\left(M_{i 1}, B_{1}\right), I\left(M_{i 2}, B_{2}\right)\right)
\end{gathered}
$$

where $I$ denotes the inclusion degree function,

$$
M_{i 1}=\left\{\left(C_{j}, \mu_{i j}, v_{i j}\right),\left(C_{k}, \mu_{i k}, v_{i k}\right), \cdots,\left(C_{p}, \mu_{i p}, v_{i p}\right)\right\}, M_{i 2}=\left\{\left(C_{s}, \mu_{b s}, v_{b s}\right)\right\}, i=1, \cdots, m
$$

Definition 9 The ranking index of alternative $M_{i}=(i=1, \cdots, m)$ is defined as follows:

$$
p_{i}=\frac{D\left(M_{i}\right)}{d\left(M_{i}\right)+D\left(M_{i}\right)},
$$

The procedure of solving multi-criteria fuzzy decision-making problem (Definition 6) is as follows:

(i) calculate the ideal solution $G_{1}$ and the anti-ideal solution $B_{1}$ satisfying the criteria $C_{j}, C_{k}, \cdots C_{p}$, calculate the ideal solution $G_{2}$ and the anti-ideal solution $B_{2}$ satisfying the criteria $C_{s}$;

(ii) calculate the inclusion degree $I\left(G_{j}, M_{i j}\right)$ of $G_{j}$ in $M_{i j}$ and the inclusion degree $I\left(M_{i j}, B_{j}\right)$ of $M_{i j}$ in $B_{j}, j=1,2 ; i=1, \cdots, m$;

(iii) calculate the inclusion degree $D\left(M_{i}\right)$ of the ideal solution in alternative $M_{i}$ and the inclusion degree $d\left(M_{i}\right)$ of $M_{i}$ in the anti-ideal solution;

(iv) calculate the ranking index $p_{i}$ of alternative $M_{i}=(i=1, \cdots, m)$

(v) if there exists $i_{0} \in\{1,2, \cdots, m\}$ such that $p_{i_{0}}=\max \left(p_{1}, p_{2}, \cdots, p_{m}\right)$, then alternative $M_{i_{0}}$ is the best choice.

The reason for introducing the ideal solution and the anti-ideal solution simultaneously in above method is that when two alternatives contain the ideal solution by the same inclusion degree, we introduce the negatively ideal solution for differentiating which alternative is superior. Then the alternative contained by the anti-ideal solution with the less inclusion degree is the better choice.

\section{An example}

Let $M_{1}, M_{2}, M_{3}, M_{4}, M_{5}$ be five alternative, and let $C_{1}, C_{2}, C_{3}$ be three criteria. Assume that the characteristics of the alternatives are represented by the IFSs shown as follows: 


$$
\begin{aligned}
& M_{1}=\left\{\left(C_{1},(0.2,0.2)\right),\left(C_{2},(0.3,0.1)\right),\left(C_{3},(0.2,0)\right)\right\} \\
& M_{2}=\left\{\left(C_{1},(0.3,0.3)\right),\left(C_{2},(0.2,0.2)\right),\left(C_{3},(0.3,0.1)\right)\right\} \\
& M_{3}=\left\{\left(C_{1},(0.4,0.4)\right),\left(C_{2},(0.5,0.4)\right),\left(C_{3},(0.3,0.2)\right)\right\} \\
& M_{4}=\left\{\left(C_{1},(0.5,0.3)\right),\left(C_{2},(0.4,0.4)\right),\left(C_{3},(0.5,0.3)\right)\right\} \\
& M_{5}=\left\{\left(C_{1},(0.4,0.4)\right),\left(C_{2},(0.6,0.3)\right),\left(C_{3},(0.6,0.4)\right)\right\}
\end{aligned}
$$

and assume that the decision-maker wants to choose an alternative which satisfies the criteria $C_{1}, C_{2}$ or which satisfies the criterion $C_{3}$.

Firstly, we construct the ideal solution and the negatively ideal solution satisfying the criteria $C_{1}, C_{2}$ and satisfying criterion $C_{3}$.

$$
\begin{aligned}
& G_{1}=\left\{\left(C_{1}, 0.5,0.2\right),\left(C_{2}, 0.6,0.1\right)\right\}, G_{2}=\left\{\left(C_{3}, 0.6,0\right)\right\} \\
& B_{1}=\left\{\left(C_{1}, 0.2,0.4\right),\left(C_{2}, 0.2,0.4\right)\right\}, B_{2}=\left\{\left(C_{3}, 0.2,0.4\right)\right\}
\end{aligned}
$$

Take $I_{3}$ in theorem 2 as the inclusion degree function $I$ and take $\lambda=\frac{1}{2}$, i.e.,

$$
I(A, B)=\frac{1}{|U|} \sum_{u \in U} \frac{R\left(\mu_{A}\left(u_{i}\right), \mu_{B}\left(u_{i}\right)\right)+R\left(v_{B}\left(u_{i}\right), v_{A}\left(u_{i}\right)\right)}{2}
$$

where we choose Lukasiewicz implication $R_{L}$ as implication $R$, i.e., $R_{L}(a, b)=\min (1-a+b, 1), \forall a, b \in[0,1]$. So we obtain the inclusion degree $I\left(G_{j}, M_{i j}\right)$ of $G_{j}$ in $M_{i j}$ and the inclusion degree $I\left(M_{i j}, B_{j}\right)$ of $M_{i j}$ in $B_{j}(j=1,2 ; i=1,2,3,4,5)$ listed in table 1 and 2

\begin{tabular}{cccccc}
\hline & $M_{11}$ & $M_{21}$ & $M_{31}$ & $M_{41}$ & $M_{51}$ \\
\hline$I\left(G_{1}, M_{\mathrm{i} 1}\right)$ & 0.85 & 0.8 & 0.825 & 0.85 & 0.875 \\
$I\left(M_{\mathrm{i} 1}, B_{1}\right)$ & 0.8 & 0.9 & 0.875 & 0.85 & 0.825 \\
\hline
\end{tabular}

Table 1: Inclusion degrees of $G_{1}$ in $M_{\mathrm{i} 1}$ and $M_{\mathrm{i} 1}$ in $B_{1}$

\begin{tabular}{llllll}
\hline & $M_{12}$ & $M_{22}$ & $M_{32}$ & $M_{42}$ & $M_{52}$ \\
\hline$I\left(G_{2}, M_{\mathrm{i} 2}\right)$ & 0.8 & 0.8 & 0.75 & 0.8 & 0.8 \\
$I\left(M_{\mathrm{i} 2}, B_{2}\right)$ & 0.8 & 0.8 & 0.85 & 0.8 & 0.8 \\
\hline
\end{tabular}

Table 2. Inclusion degrees of $G_{2}$ in $M_{\mathrm{i} 2}$ and $M_{\mathrm{i} 2}$ in $B_{2}$

Using formulae (5) and (6) we get the inclusion degree $D\left(M_{i}\right)$ of the ideal solution in alternative $M_{i}$ and the inclusion degree $d\left(M_{i}\right)$ of $M_{i}$ in the negatively ideal solution. We fist them in table 3.

\begin{tabular}{cccccc}
\hline & $M_{1}$ & $M_{2}$ & $M_{3}$ & $M_{4}$ & $M_{5}$ \\
\hline$D\left(M_{\mathrm{i}}\right)$ & 0.85 & 0.8 & 0.825 & 0.85 & 0.875 \\
$d\left(M_{\mathrm{i}}\right)$ & 0.8 & 0.8 & 0.85 & 0.8 & 0.8 \\
\hline
\end{tabular}

Table 3: Inclusion degrees of $G_{2}$ in $M_{\mathrm{i} 2}$ and $M_{\mathrm{i} 2}$ in $B_{2}$

From (7), we get the ranking index of alternatives as follows:

$$
p_{1}=0.515, p_{2}=0.5, p_{3}=0.4925, p_{4}=0.515, p_{5}=0.5224
$$

Therefore, alternative $M_{5}$ is the best choice.

\section{Conclusion}

This paper presents a fuzzy optimization method based on the inclusion degrees of intuitionistic fuzzy sets to solve multi-criteria decision making problems under fuzzy environments. Inclusion degree is a quantity describing that a set is contained by another set and is quantitative description of containment relation. It holds the uncertainty of the relation. The inclusion degree theory and IFS theory are the important tools in studying the uncertain knowledge. The proposed method in the paper has been demonstrated by an example, illustrating the power of the approach to solve multi-criteria fuzzy decision making problems. This research work not only develops and enriches the fundamental theory of IFSs but also provides a new idea 
for the applications of IFS theory.

\section{Acknowledgement}

This research work is supported by the Natural Science Fund of China (\# 70501022).

\section{References}

[1] Zadeh L. A. Fuzzy sets. Information and Control 1965; 8(3): 338 - 356.

[2] Bortolan G., Degani R. A review of some methods for ranking fuzzy subsets. Fuzzy Sets and Systems 1985; 15(1) (1985) pp: 1-19.

[3] Liou T. S., Wang M. J. Ranking fuzzy numbers with integral value. Fuzzy Sets and Systems 1992; 50(3): 247 255.

[4] Tran L. and Duckstein L. Comparison of fuzzy numbers using a fuzzy distance measure. Fuzzy Sets and Systems 2002; 130(3): 331 - 341.

[5] Wang X. Z. and Kerre E.E. Reasonable properties for the ordering of fuzzy quantities (I) and (II). Fuzzy Sets and Systems 2001; 118(3): $387-405$.

[6] Atanassov K. Intuitionistic fuzzy sets, VII ITKR’s Session, Sofia, June 1983 (Deposed in Central ScienceTechnical Library of Bulg. Academy of Science, 1697/84) (in Bulgarian).

[7] Chen S. M. and Tan J. M. Handling multi-criteria fuzzy decision-making problems based on vague set theory. Fuzzy Sets and Systems 1994; 67(2): 163-172.

[8] Hong D.H.and Choi C.H. Multi-criteria fuzzy decision-making problems based on vague set theory. Fuzzy Sets and Systems 2000; 114: 103-113.

[9] Szmidt E. and Kacprzyk J. Group decision making under intuitionistic fuzzy preference relations. Proc. of the Seventh International Conference on IPMU 1998; 172-178.

[10] Atanassov K., Pasi G. and Yager R. Intuitionistic fuzzy interpretations of multi-measurement tool multi-criteria decision making. Proc. of the Sixth International Conference on Intuitionistic Fuzzy Sets, Varna, 13-14 September 2002, Lecture Notes on Intuitionistic Fuzzy Sets 2002; 8(3): 66-74.

[11] Atanassov K., Pasi G., Yager R.and Atanassova V. Intuitionistic fuzzy group interpretations of multi-person multicriteria decision making. Proc. of the Third Conference of the European Society for Fuzzy Logic and Technology EUSFLAT’2003. Zittau, 10-12 September 2003; 177-182.

[12] Pasi G., Yager Y.and Atanassov K. Intuitionistic fuzzy graph interpretations of multi-person multi-criteria decision making: Generalized net approach. Proc. of 2004 second International IEEE Conference Intelligent Systems 2004; 2: 434-439.

[13] Atanassov K. Intuitionistic fuzzy sets. Fuzzy Sets and Systems 1986; 20: 87 - 96.

[14] Atanassov K. Intuitionistic fuzzy sets. Physica-Verlag, Heidelberg, New York, 1999.

[15] Fan J., Xie W.and Pei J. Subsethood measure: new definitions. Fuzzy Sets and Systems 1999; 106: 201-209.

[16] Chen C. T. Extensions of the TOPSIS for group decision-making under fuzzy environment. Fuzzy Sets and Systems 2000; 114(1): 1-9.

[17] Chu T. C.and Lin Y. C. A fuzzy TOPSIS method for robot selection. The International Journal of Advanced Manufacturing Technology 2003; 21: 284-290.

[18] Wang Y. M.and Elhag T. Fuzzy TOPSIS method based on alpha level sets with an application to bridge risk assessment. Expert Systems with Applications 2006; 31(2): 309 - 319.

[19] Abo-Sinna M. A.and Amer A. H. Extensions of TOPSIS for multi-objective large-scale nonlinear programming problems. Applied Mathematics and Computation 2005; 162(1): 243 - 256.

[20] Liang G. S. Fuzzy MCDM based on ideal and anti-ideal concepts. European Journal of Operational Research 1999; 112(3): $682-691$. 